\title{
Report: State-of-the-Art DE-ghosting Algorithms for High Dynamic Range Imaging
}

\author{
Kanita Karađuzović-Hadžiabdić, Jasminka Hasić Telalović \\ International University of Sarajevo, Faculty of Engineering and Natural Sciences, HrasnickaCesta 15, \\ Ilidža71210 Sarajevo, Bosnia and Herzegovina
}

\author{
Article Info \\ Article history: \\ Keywords: \\ High dynamic range image, deghosting \\ algorithms, ghost removal
}

\begin{abstract}
High Dynamic Range Imaging (HDRI) may be generated by combining multiple low dynamic range (LDR) images of the same scene with different exposures. However, this technique of generating HDR images is limited to static scenes. During capturing of LDR images, any changes in the scene between different captures caused by either camera shake or object motion results in ghost artifacts in the produced HDR image. These ghost artifacts make the same object appear more than once in the generated HDR image. As a result, deghosting algorithms have been developed over the past years in an attempt to remove any ghosting present in HDR images.This article is a report of „State-ofthe-art deghosting algorithms for generating HDR images“(Karađuzović-Hadžiabdić K., and Hasić Telalović J., 2013) .
\end{abstract}

\section{INTRODUCTION}

Mainstream consumer cameras capture only 8-bit images per color channel per pixel. This does not cover the full dynamic range of irradiance values present in most real world scenes.Several methods exist of capturing high dynamic range (HDR) illumination present in typical real world scenes:

- Specialized hardware

- $\quad$ Special CCD design to obtain HDR imaging, e.g. using multiple sensors with different sensitivity

- Commercially only a few companies Panoscan Mark3, SpheronVRmanifacture HDR cameras, but are extremely expensive.

Most common way of producing HDR images is by capturing a sequence of low dynamic range (LDR) images of the same scene with different exposures and combining those to produce an HDR image (Debevec and Malik 1997).
Multi exposure approach produces high quality HDR images only for static scenes taken on a tripod.Any change in the scene in between each capture of LDR images or any slightest camera motion results in ghost artifacts in the resultant HDR image. As a result, over the past years a number of algorithms wasdesigned to deal with ghost removal in HDR images.

LCD monitors cannot display all of the dynamic range stored in the HDR images since dynamic range of these devices are limited.To solve this problem, tone mapping (TM) is applied to HDR image prior to display.TM operators reduce the dynamic range of the input image to fit the dynamic range of the display.

\section{State-of-the-art-deghosting-methods}

State-of-the-art deghosting algorithms for high dynamic range imaging (Karađuzović-Hadžiabdić and HasićTelalović, 2013) providesan overview of 5 state-of-the-art deghosting algorithms in HDR imaging.The following gives an overview of these algorithms. The name of the method in italic letters is the name of the algorithm that will be referred to throughout this paper. 


\section{Patch-Based HDR Reconstruction:(Sen et al. 2012)}

Sen et al. (Sen 2012) algorithm is a patch-based energyminimization formulation. The algorithm produces an HDR image from a set of LDR images captured with different exposures, that is aligned to the reference image $\mathrm{L}_{\text {ref. }}$ which is an LDR image that contains most well exposed pixels. The resultant HDR image contains as much information as possible from the well-exposed pixels from the $\mathrm{L}_{\text {ref }}$.In places where $\mathrm{L}_{\mathrm{ref}}$ is not well exposed, every patch in the image $\mathrm{H}$ at a given exposure should have a similar patch in one of the LDR images after exposure adjustment (coherence). Also, every exposureadjusted patch in all $\mathrm{L}_{\mathrm{k}}$ images should be contained in $\mathrm{H}$ at exposure $\mathrm{k}$ (completeness). The iterative approach performs joint optimization of image alignment and HDR merge process until all the exposures are correctly aligned to the reference exposure and a good quality HDR result is produced.

HDR generation from exposure stacks (Hu et al. 2013) :

This is also a patch-based algorithm that uses an iterative approach to register LDR images to a reference image. The algorithm produces a registered stack from a sequence of misaligned images of dynamic scenes. Initially, the reference image $\mathrm{R}$ is selected, then, for each source image in the exposure stack, the algorithm synthesized a new image $\mathrm{L}$ that looks like the reference image R, exposed like S. Image L, has the image content geometrically compatible with $\mathrm{R}$, wherever $\mathrm{R}$ is well exposed. The algorithm propagates the intensity and gradient information during HDR reconstruction. It performs well even if the reference image has large saturated areas.

Optical flow based algorithm: (Zimmer et al. 2011):

Zimmer et al (2011) use state-of-the-art optical flow approach to register LDR exposures before the merging process. They minimize their proposed energy function that uses a data term and smoothness term to reconstruct saturated and occluded areas.After alignment, the displacement fields obtained with subpixel precision are used to produce a super resolved HDR image.

Photoshop CS5 Extended, version 12.0 (Photoshop) and Photomatix Pro (2012), version 4.2.6 (Photomatix): details of the implementation of deghosting algorithms for implemented in these two commercial softwares are not publicaly available.

\section{METHODOLOGY}

To test the mentioned state-of-the-art algorithms we used a wide range of the scenes as algorithm performance may be scene dependent.Selection of the scenes were based following the criteria proposed by Baker et al. (2011).

As a result, the dataset used consists of variety of real-world scenes with: fast and abrupt motion, high textured motion, independently moving objects, scenes taken with a hand held camera, occlusions, large motion displacement, small motion displacement and stereo sequences of a static scene.
A sequence of three LDR images with different exposures were captured by a digital Canon EOS 1000D camera.

To present the HDR image on an LDR display tone mapping needs to be used, which may hide the artifacts of the deghosting algorithms.We used 'gamma correction' $(\gamma=2.2)$ contrast compression, which mimics the response of a typical LCD display and $p f$ sview HDR image viewer from the pfstools package (Mantiuk2007) to produce .png images. The resultant image resolution was $1360 \times 758$.

\section{RESULTS AND DISCUSSION}

This section outlines the results of each individual scene:

Complex motion discontinuity scene: Table 1. displays HDR images of 5 mentioned state-of-the-art algorithms for a complex dynamic scene containing several independently moving objects, large motion displacement and occlusion.As it can be seen from Table 1.Photomatix and Zimmer produced well visible artifacts. Hu2013 also produced some less visible artifacts at the edges of the man's legs. No artifacts were observed for Photoshop and Sen2012 methods.

Static plant hand held camera scene:Table 2 displays the images of a static scene of a green plant. Photomatix produced artifacts in the form of dark splotches on the frame of the window (see Figure 1). No artifacts can be seen for other algorithms.

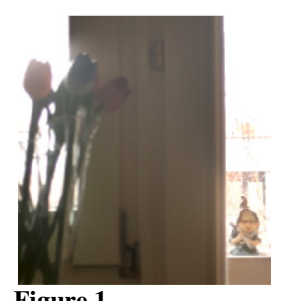

Figure 1.

High texture motion scene: Table 3. shows HDR images of a scene that includes high texture motion and small motion displacement. Zimmer2011 and Photoshop algorithms produced artifacts at the moving leaves of the tree (see Fig. 2.). No visible artifacts are visible for other algorithms.
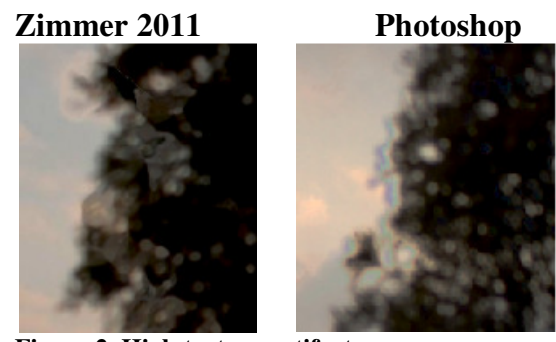

Figure 2. High texture artifacts 
Independently moving objects scene: Table 4. shows HDR images of a scene that contains independently moving objects. Only Zimmer2011 produced artifacts visible at the edges of the vase (see Fig 3.). Other algorithms did not produce any visible artifacts.

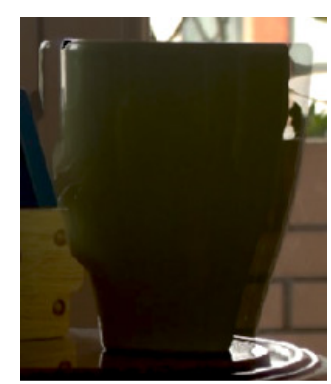

Fig 3. Artifacts produced by Zimmer2011 method.

Abrupt motion scene: Table 5. shows HDR images of a complex dynamic scene involving significant and fast abrupt motion, large motion displacement and occlusion. Zimmer2011 and Photoshop method produced artifacts that are visible in Table 5. Other algorithms performed reasonably well.

Stereo and occlusion scene: Table 6. shows HDR images of a stereo sequence of a static scene. All of the algorithms failed to produce artifact free images. Figure 4.shows the artifacts present for the images produced by Sen2012, Hu2013 and Photomatix. Sen 2012 algorithm produced an image that contains a new texture on the window area that is not present in the real scene. Hu2012 has well visible artifacts shown on the edges of the tulips and the window frame. Photomatix algorithm generated gay areas in the window area, and there is a visible loss of contrast on tulips. Photoshop produced the best results were artifacts are hardly visible.

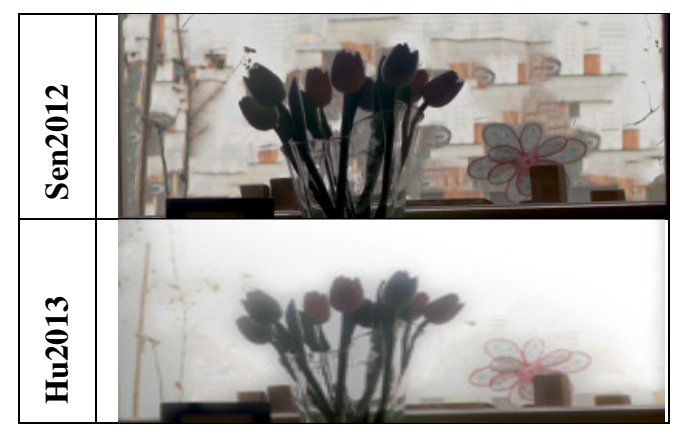

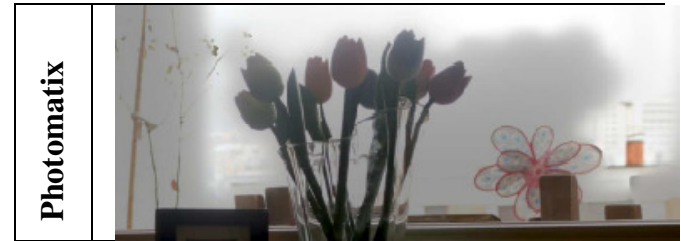

Figure 4. Artifacts produced by Sen2012, Hu2013 and Photomatix method for the Stereo and occlusion scene.

TABLE 1.

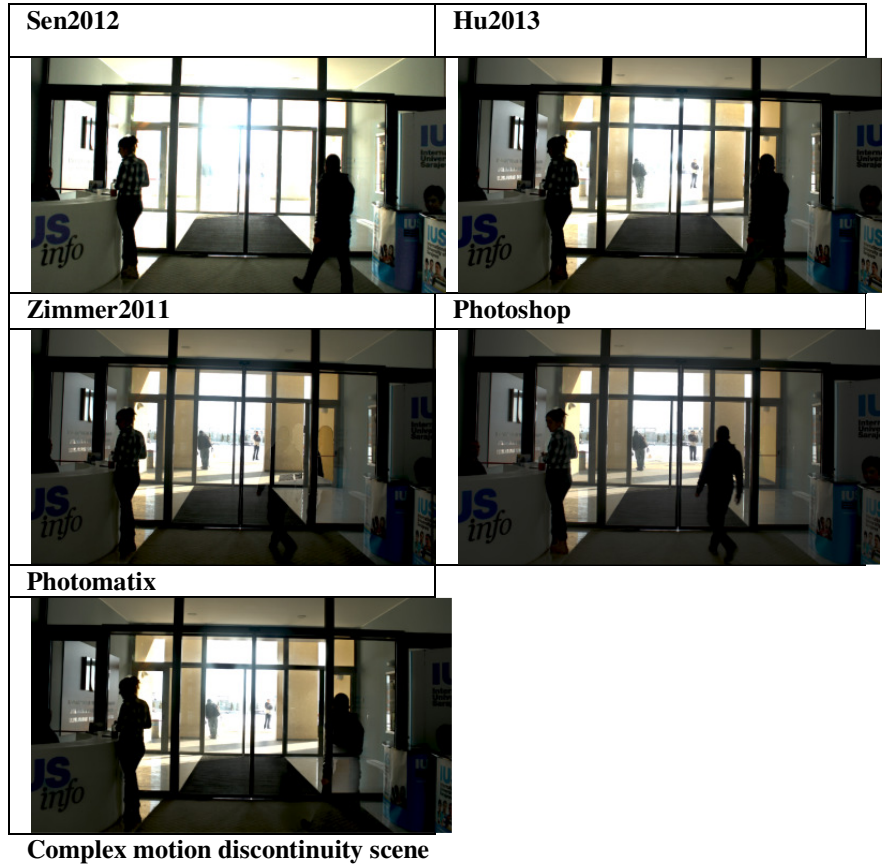

TABLE 2.

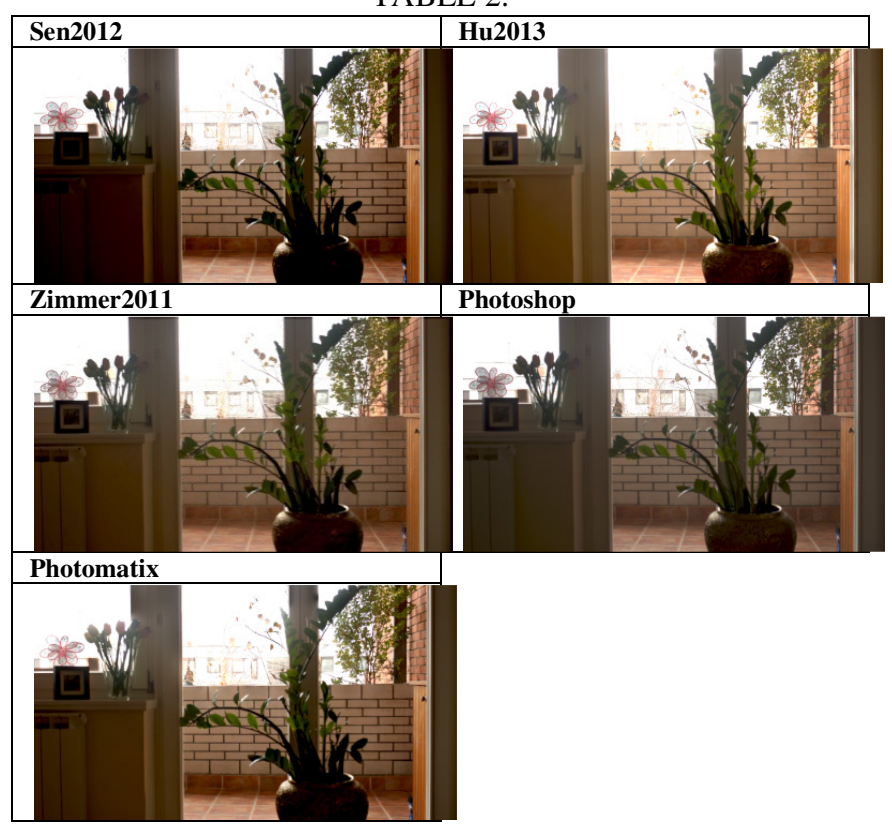


Static plant hand held camera scene captured with handheld camera using automatic exposure bracketing $(-2 \mathrm{EV}, 0 \mathrm{EV}, 2 \mathrm{EV})$

TABLE 3.

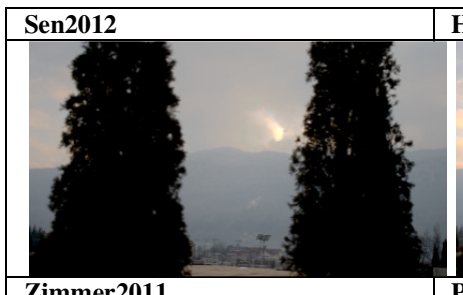
Hu2013

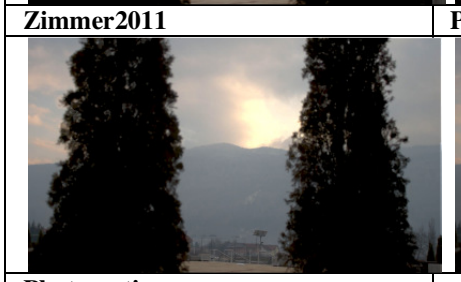
Photoshop

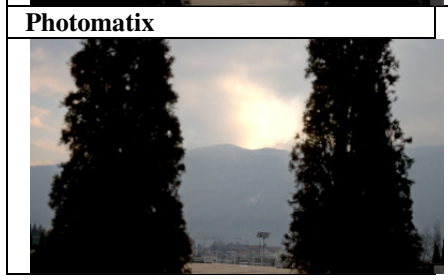

High texture motion scene: evergreen trees were moved between LDR capture to simulate motion.

TABLE 4.
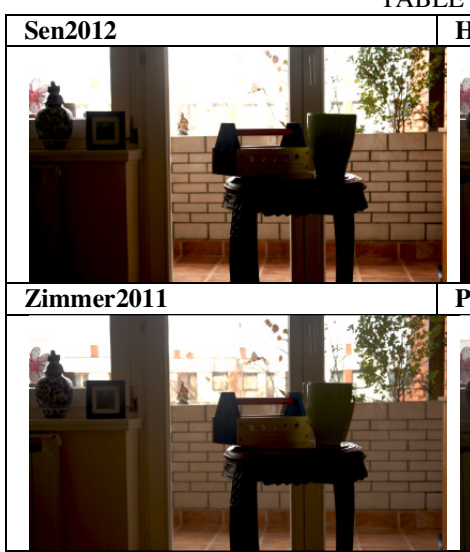

Photoshop
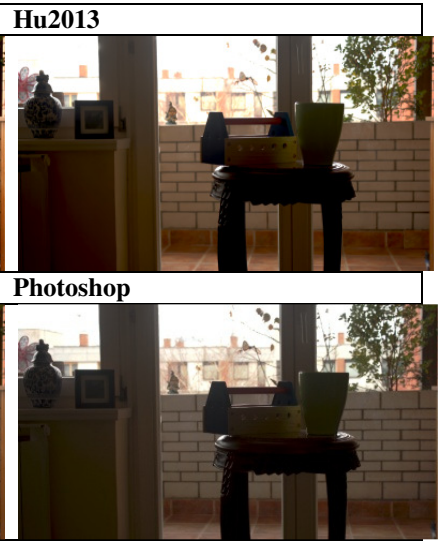

Photomatix

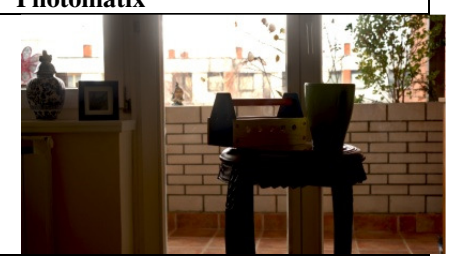

Independently moving objects scene: objects were moved between LDR capture to simulate motion.
TABLE 5.
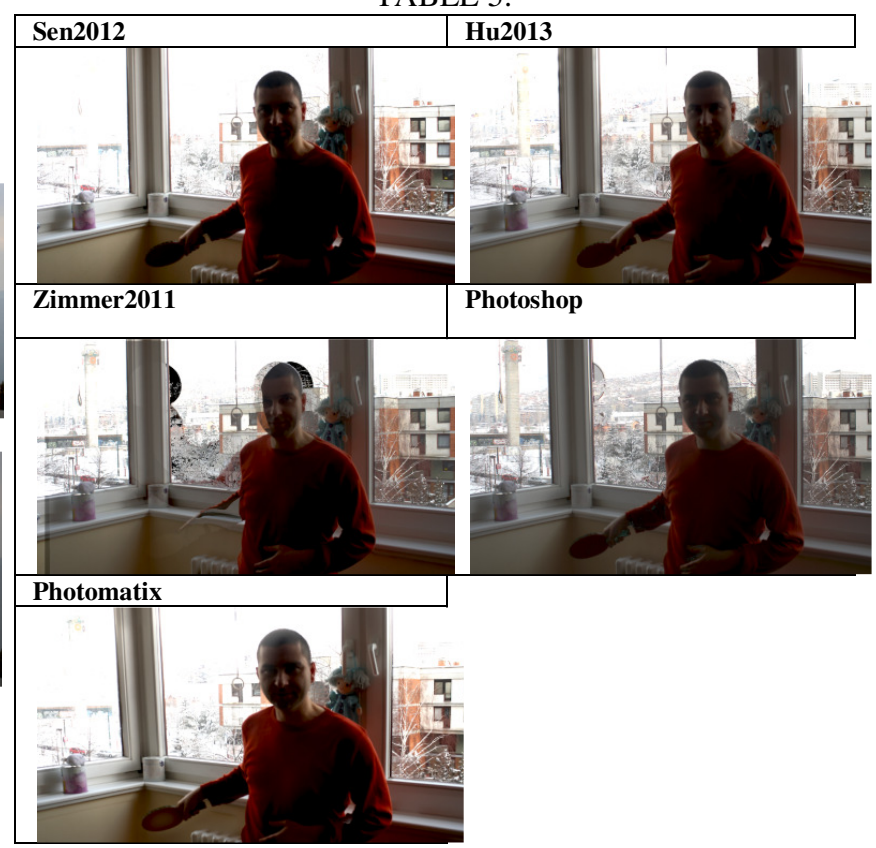

Abrupt motion scene

TABLE 6.

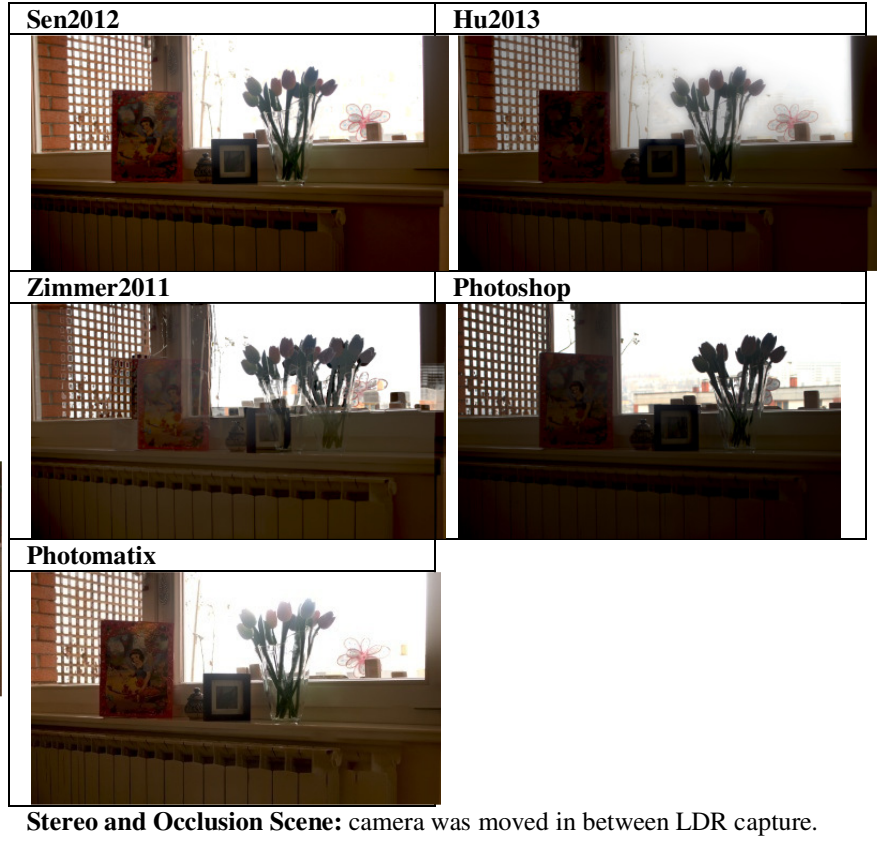

\section{CONCLUSIONS}

Wide range of dynamic scenes used to test the performance of 5 state-of-the-art algorithms.

- Sen2012 and Hu2013methods, that are patch-based and use iterative approach to reconstruct HDR image produce least artifacts. 


\section{ACKNOWLEDGMENT}

- Zimmer2011 based on optical flow estimation performs well for scenes that involve small continuous motion, but not for scenes with large displacements and abrupt motion discontinuities.

- Photoshop (commercial software): we observed that Photoshop fully or partially ignores the exposures that are not aligned to the reference image.

We would like to thank Henning Zimmer and Hu et. al for running their code on our datasets. This work was partly supported by COST Action IC1005 "HDRi The digital capture, storage, transmission and display of real-world lighting".

\section{REFERENCES}

Baker, Simon and Scharstein, Daniel and Lewis, J.P. and Roth, Stefan and Black, Michael J. and Szeliski, Richard (2011), " A Database and Evaluation Methodology for Optical Flow", International Journal of Computer Visio $92 n$,

Debevec, P., Malik, J., (1997) "Recovering high dynamic range radiance maps from photographs", In Proceedings of the SIGGRAPSH '97 Conference, 1997, pp 369-378

Hu, Jun., Gallo, O., Pulli, K., and Sun, X,. "HDR Deghosting: How to deal with Saturation?", (2013) InProceedings of the IEEE Conference on Computer Vision and Pattern Recognition, June 2013

Karađuzović-Hadžiabdić K, and HasićTelalović J. (2013), "State-of-the-art deghosting algorithms for high dynamic range imaging",European Conference in Technology and Society, (EuroTecS 2013), 27-28 June, 2013

Mantiuk, Rafal and Krawczyk, Grzegorz and Mantiuk, Radoslaw and Seidel, Hans-peter, (2007), "High dynamic range imaging pipeline perception-motivated representation of visual content ", In Human Vision and Electronic Imaging, B. E. Rogowitz, T. N. Pappas, and S. J. Daly, Eds., no. Setion 2, 649212-649212-12

Photomatix, (2012), Commercially-available HDR processing software \}, http://www.hdrsoft.com

Sen, P., Kalantari, N. K., Yaesoubi, M., Darabi, S., Goldman, D. B., and Shechtman, E. (2012) Robust patchbased hdr reconstruction of dynamic scenes.", In Proceedings of SIGGRAPSH Asia 2012, ACM Transactions on Graphics, 31(6), 203:1-203:11
- Photomatix (commercial software): we observed that algorithm uses spatially adaptive weighting of exposures, that my result in splotchy artifacts.

Zimmer, H., Bruhn, A., and Weickert, J. (2011), "Freehand HDR imaging of moving scenes with simultaneous resolution enhancement." In Proceedings of Eurographics Computer Graphics Forum, 30(2), pp. 405414 